\title{
Aspectos teóricos sobre la biofísica de la radiofrecuencia aplicada al tratamiento del dolor
}

\author{
V. Muñoz ${ }^{1}$ J.J. Pérez², J.J. Pérez-Cajaraville ${ }^{3}$ y E. Berjano ${ }^{4}$ \\ ${ }^{I}$ Neurotherm Spain. Barcelona. ${ }^{2}$ Instituto de Investigación Interuniversitario en Bioingeniería y Tecnología \\ Orientada al Ser Humano. Universitat Politècnica de València. Valencia. ${ }^{3}$ Pain Unit and Department \\ of Anesthesia and Critical Care. Clínica Universidad de Navarra. Universidad de Navarra, Pamplona. \\ ${ }^{4}$ Biomedical Synergy. Electronic Engineering Department. Universitat Politècnica de València. Valencia
}

Muñoz V, Pérez JJ, Pérez-Cajaraville JJ, Berjano E. Aspectos teóricos sobre la biofísica de la radiofrecuencia aplicada al tratamiento del dolor. Rev Soc Esp Dolor 2014; 21(6): 351-358.

\begin{abstract}
Interventional techniques for pain treatment have importantly increased in recent years, and particularly those based on radiofrequency energy, both thermal and pulsed. This report clarifies some important concepts about electrical phenomena involved during using these techniques, given a comprehensive explanation about types and modes of applying RF energy. Moreover, guidelines about the patterns of affected zones are defined dependent on the chosen arrangement. From a physical point of view, equations governing RF process are also presented. RF generator users should be knowledgeable about what is happening in each RF-based treatment in order to select the most suitable arrangement for the patient.
\end{abstract}

Key words: Radiofrequency. Thermal. Pulsed. Theoretical aspects. Morphology. Radiofrequency type. Radiofrequency mode. Government equations.

\section{RESUMEN}

Las técnicas intervencionistas para el tratamiento del dolor han experimentado en los últimos años un significativo aumento, y particularmente las basadas en energía de radiofrecuencia, tanto térmica, como pulsada. Este artículo clarifica algunos de los principales conceptos relacionados con los fenómenos eléc-

Recibido: 05-09-14.

Aceptado: 15-11-14 tricos que ocurren durante el uso de las mencionadas técnicas, proporcionando una comprensión sobre los tipos y modos existentes de aplicación de radiofrecuencia. Asimismo, se definen las pautas en la morfología de las zonas afectadas según las distintas configuraciones, y se revisa, desde un punto de vista físico, cuáles son las ecuaciones que aplican en cualquier proceso de radiofrecuencia. El usuario de los generadores de radiofrecuencia debe tener conocimientos sobre lo que está ocurriendo en cada uno de los posibles tratamientos, con el objeto de seleccionar la más idónea de las opciones para el paciente.

Palabras clave: Radiofrecuencia. Térmica. Pulsada. Aspectos teóricos. Morfología. Tipos de radiofrecuencia. Modos de radiofrecuencia. Ecuaciones de gobierno.

\section{INTRODUCCIÓN}

La utilización de las corrientes eléctricas de radiofrecuencia es una técnica clínica ampliamente extendida en el ámbito del tratamiento del dolor crónico $(1,2)$. Se trata de una técnica mínimamente invasiva, de acceso percutáneo en la mayoría de los casos, que consiste en la aplicación de un campo eléctrico de radiofrecuencia (en torno a $500 \mathrm{kHz}$ ) alrededor de un tejido, a través de un aplicador, que produce una modificación en el tejido diana tratado, y consecuentemente una alteración en la transmisión del estímulo doloroso.

Los componentes necesarios de un sistema de radiofrecuencia son: un generador de radiofrecuencia, capaz de emitir energía a la frecuencia, y modularla en función de los parámetros definidos (3) (Fig. 1). Actualmente existen en el mercado generadores tecnológicamente avanzados capaces de suministrar la energía a través de múltiples canales y con gran cantidad de funciones de parametrización y respaldo de datos. 


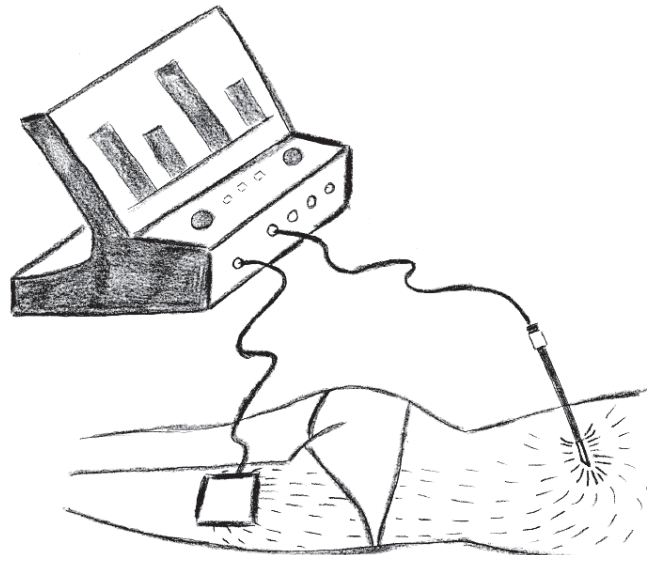

Fig. 1. Esquema eléctrico basado en un generador, electrodo, aguja y placa de dispersión.

El electrodo de radiofrecuencia es el elemento a través del cual se transmite la energía eléctrica hasta el aplicador distal. El electrodo también incluye un sensor de temperatura que sensa dicha magnitud y proporciona al generador esta información.

El aplicador o componente distal es el elemento que entra en contacto con el cuerpo humano. Habitualmente es una aguja de radiofrecuencia u otro tipo de electrodo o catéter. La punta activa del aplicador, también llamada polo/s activo/s, es la zona metálica que queda expuesta, y a través de la cual se distribuye la radiofrecuencia al cuerpo humano. El resto del aplicador suele estar recubierto de material aislante. La placa de dispersión que se adhiere a la piel del paciente es el elemento que permite que se cierre el circuito eléctrico y proporciona por lo tanto un retorno de energía hasta el generador de radiofrecuencia.

Actualmente la energía de radiofrecuencia utilizada en el ámbito del tratamiento del dolor se basa en una onda senoidal de frecuencia aproximada a los $500 \mathrm{kHz}$, la cual corresponde a 500.000 ciclos por segundo. Esto es 500.000 cambios de polaridad en un segundo. Es necesario conocer que cuando dicha energía atraviesa el tejido diana produce un aumento local de la temperatura debido a la agitación iónica. Dicho aumento de temperatura se transmite a la punta o polo activo del aplicador desde el tejido circundante, y es registrada por el sensor de temperatura situado en el mismo. La monitorización de este valor de temperatura es utilizada por el generador para modular la emisión de energía en el caso de que sea necesario.

\section{MODOS DE RADIOFRECUENCIA}

Existen dos modos de radiofrecuencia, la térmica y la pulsada.

La radiofrecuencia térmica, también denominada radiofrecuencia continua, convencional, lesiva o ablativa (CRF), se basa en la aplicación de energía de radiofrecuencia en forma continua con el fin de causar una lesión en los tejidos que circundan al polo activo (4). En este modo de funcionamiento existen parámetros controlables, tales como la temperatura y la impedancia, los cuales determinan la modulación de la salida de energía (Fig. 2).

En la práctica habitual, y para comprender esta definición, se define una temperatura objetivo y un tiempo de tratamiento o lesión. Al iniciar el procedimiento el generador de radiofrecuencia aplica un valor alto de voltaje con el fin de alcanzar lo antes posible la temperatura objetivo desde la temperatura basal del paciente siguiendo un perfil de rampa ascendente, medida en grados/segundo, y establecido por cada fabricante (Fig. 2B). Una vez alcanzada la temperatura objetivo en el polo activo, el generador de radiofrecuencia modula la salida de energía para poder mantener la temperatura objetivo durante el tiempo establecido de lesión. Hasta que no se alcanza la temperatura objetivo, no empieza a descontar el tiempo de lesión. Por lo tanto se trata de un tiempo de lesión efectivo. La modulación realizada por los generadores de radiofrecuencia varía en función de cada modelo de generador o fabricante, siendo estas o bien la modulación a picos, o bien la modulación constante a bajas amplitudes. La modulación a picos se entiende como la emisión de pulsos de alta amplitud y de poca duración, con lo que podría entenderse como una pulsatilidad continua o "pseudocontinua". La modulación

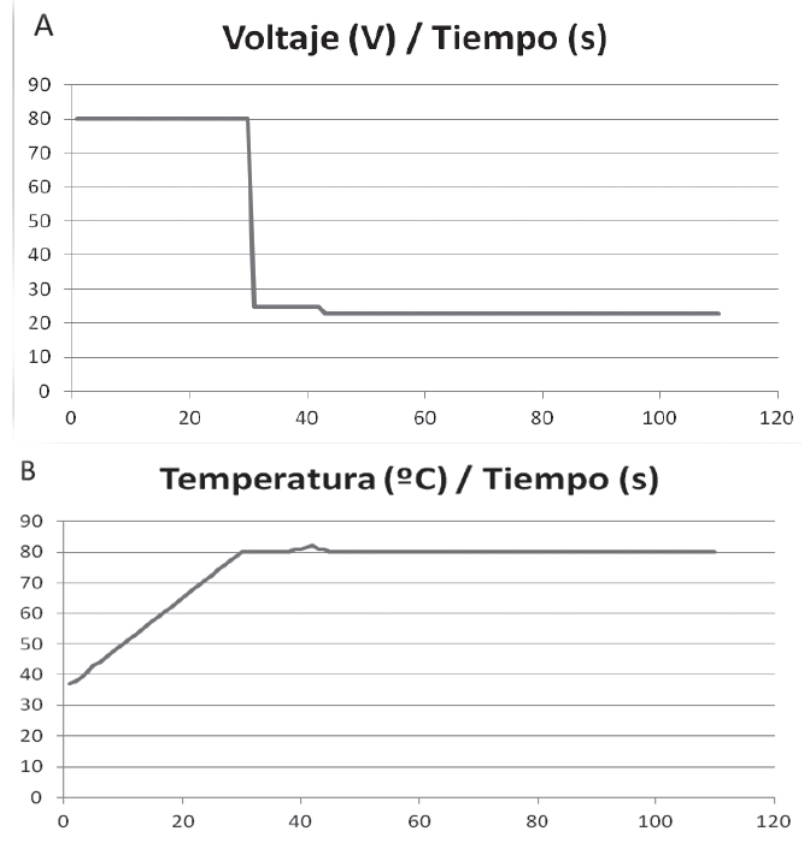

Fig. 2. Gráficos de voltaje aplicado (A) y temperatura detectada en el polo activo (B) durante un procedimiento de radiofrecuencia térmica. 
constante pretende emitir amplitudes bajas moduladas pero continuas (Fig. 2A). En ambos casos se cumple con el propósito de mantenimiento de la temperatura objetivo, siendo $80{ }^{\circ} \mathrm{C}$ para radiofrecuencia térmica, y un máximo de $42{ }^{\circ} \mathrm{C}$ para radiofrecuencia pulsada. No existen estudios que demuestren ventajas e inconvenientes de las distintas posibilidades de modulación de la amplitud.

Durante este modo de funcionamiento se monitoriza, además de la temperatura, la evolución de la impedancia. Durante la aplicación de la radiofrecuencia, la impedancia tiende a disminuir debido al efecto térmico, el cual hace aumentar la conductividad eléctrica y térmica de los tejidos diana. Se trata simplemente de un parámetro de control que afecta al procedimiento en caso de alcanzar valores extremos, tanto por arriba como por abajo. En el caso de que la medida de impedancia llegara a 0 ohmios (Fig. 3), el generador de radiofrecuencia paralizaría automáticamente la salida de energía por entender que existe un circuito abierto. Por el contrario, cuando la medida de impedancia supera un valor máximo establecido (variable según fabricantes), el generador paraliza la emisión de energía por entender que se ha creado una cápsula de tejido vaporizado que rodea por completo el polo y que puede estar derivado de alguna complicación de la técnica, tales como problemas de medición de temperatura en el caso de CRF. En este caso la lesión no alcanza el tamaño deseado.

La radiofrecuencia pulsada (PRF) (5-8) consiste en la aplicación de pulsos de potencia de radiofrecuencia cada cierto periodo, separados por periodos de tiempo más largos en los cuales no se aplica potencia. Aunque la duración de los pulsos es parametrizable, corresponde siempre a un porcentaje muy bajo del total del periodo. Esto es, si el periodo es de 1 segundo, y la duración del pulso es de $50 \mathrm{~ms}$, significa que el porcentaje de aplicación de potencia es de 0,005, es decir, del $5 \%$.

El objetivo de la radiofrecuencia pulsada es que el tejido no alcance un valor de temperatura correspondiente a

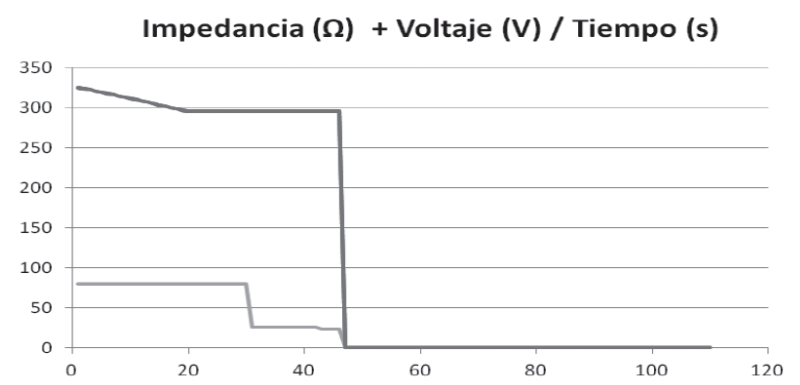

Fig. 3. Gráfico de impedancia en simulación de circuito abierto, donde se deja de emitir energía al medir impedancia $=0$. una lesión ablativa. Es por ello que utiliza este modo de funcionamiento para depositar alrededor de la punta activa de la aguja/polo un campo eléctrico que cumple una función neuromoduladora de la transmisión del estímulo doloroso. Existen diversas teorías que determinan el principio de acción de la radiofrecuencia pulsada (9-11). No es el objeto del presente documento abordar dichas teorías. En radiofrecuencia pulsada se pueden modificar o variar los siguientes parámetros (Fig. 4):

- Voltaje aplicado: determina la amplitud del pulso emitido. Cuanto mayor es el voltaje, mayor es la cantidad del campo eléctrico aplicado en el tejido.

- Anchura de pulso: determina la duración durante la cual el voltaje es aplicado.

- Frecuencia de repetición de los pulsos: determina la cantidad de pulsos emitidos por cada segundo, por ejemplo, una frecuencia de $2 \mathrm{~Hz}$ implica dos pulsos por segundo. No confundir esta frecuencia con la frecuencia de la señal senoidal de radiofrecuencia (habitualmente de $500 \mathrm{kHz}$ ) que es la que se emite durante el tiempo que dura cada pulso.

- Temperatura límite: determina el valor de la temperatura medida por el electrodo a partir de la cual el generador de radiofrecuencia actúa con el fin de que la temperatura no supere dicho valor, y por lo tanto se cree una lesión ablativa. La actuación del generador puede basarse o bien en la supresión de algunos pulsos o la reducción automática del voltaje o ancho de pulso aplicado.

- Tiempo de tratamiento: es el tiempo total programado.

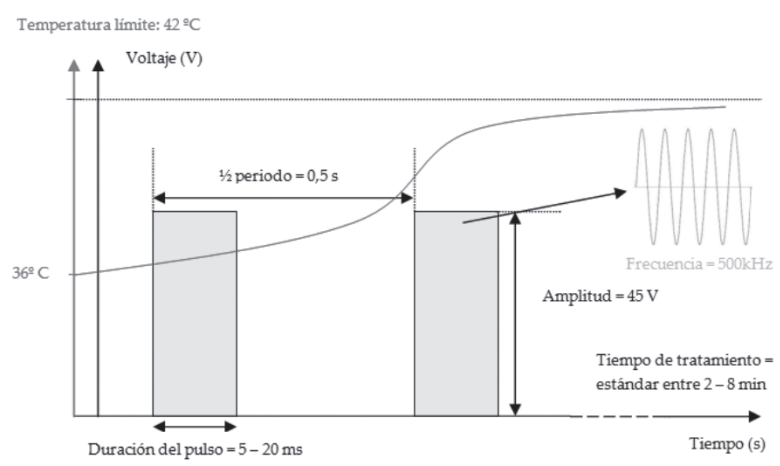

Fig. 4. Gráfico de modo de radiofrecuencia pulsada.

Al igual que en el modo de funcionamiento de radiofrecuencia térmica, durante el modo de radiofrecuencia pulsada también se monitoriza, además de la temperatura, la impedancia eléctrica. Se trata simplemente de un parámetro de control, que afecta al procedimiento si se miden valores extremos, tanto por superiores como inferiores. 


\section{Definición de pulsada dosis}

El modo de funcionamiento de pulsada dosis es un modo único, y patentado por Neurotherm (Wilmington, MA, USA), donde el parámetro tiempo de tratamiento no es un intervalo temporal sino un número de pulsos de tratamiento. Se obtiene simplemente multiplicando la frecuencia de repetición de los pulsos por el tiempo de tratamiento. Durante un procedimiento tradicional de radiofrecuencia pulsada, cuando la temperatura medida en el polo activo alcanza la temperatura límite definida por el operador, el generador de radiofrecuencia comienza a modular la emisión de los pulsos con el fin de no sobrepasar dicha temperatura límite (Fig. 5). Pero mientras esto ocurre, el tiempo de tratamiento sigue corriendo y por lo tanto el número de pulsos aplicado será menor en el caso de que ocurra esta modulación. Así, en los posibles casos de pobre disipación térmica donde la temperatura del polo activo sube excesivamente, del tiempo total de tratamiento, habrá un porcentaje durante el cual no se emiten pulsos, o se emiten pulsos de menor voltaje, o con menor ancho de pulso que el parametrizado (Fig. 6). La consecuencia es que la dosis eléctrica programada (número de pulsos a determinado voltaje) no es realmente aplicada al tejido.

En el modo de funcionamiento de pulsada dosis, el tiempo de tratamiento se convierte en un número efectivo de pulsos de tratamiento. Para ello, el generador de radiofrecuencia aumenta en caso necesario el tiempo del periodo silente (periodo entre pulso y pulso) (Fig. 7), con el objeto de que la temperatura medida en la punta activa disminuya, y cuando se haya situado por debajo de la temperatura límite, vuelva a lanzar pulsos de voltaje y duración de pulso parametrizados (programados). De esta manera, se garantiza que tanto el número total de pulsos como su voltaje son aquellos definidos por el operador.
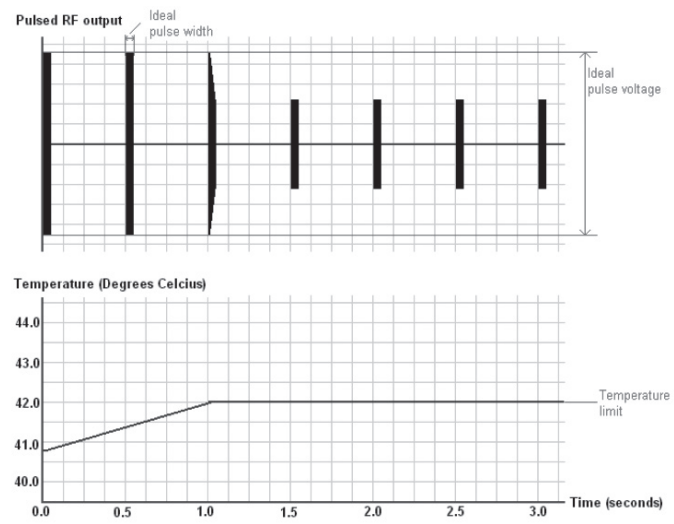

Fig. 5. Gráfico de modo de radiofrecuencia pulsada dosis, donde al alcanzar la temperatura límite, el generador modula el voltaje emitido.
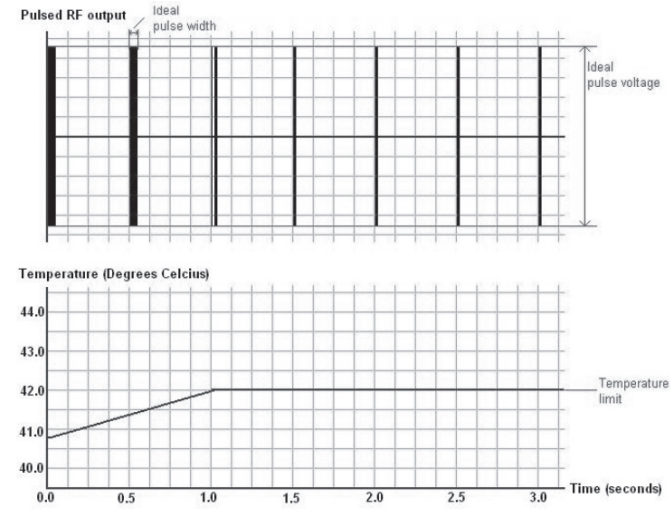

Fig. 6. Gráfico de modo de radiofrecuencia pulsada dosis, donde al alcanzar la temperatura límite, el generador modula el ancho de pulso emitido.
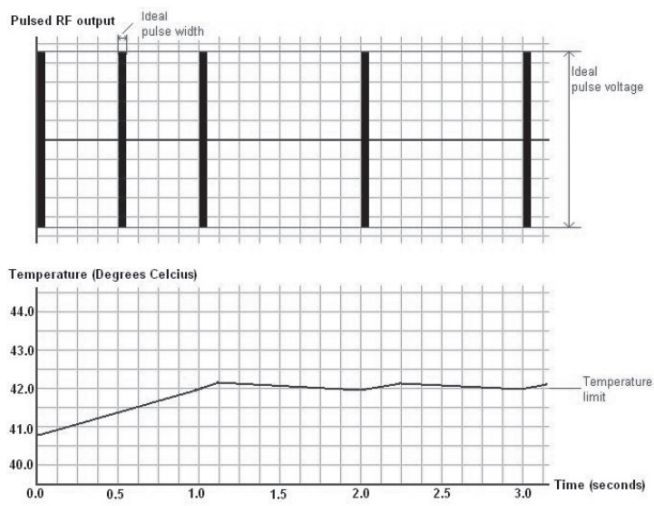

Fig. 7. Gráfico de modo de radiofrecuencia pulsada dosis, donde al alcanzar la temperatura límite, el generador aumenta el periodo silente sin necesidad de modular el voltaje ni el ancho de pulso emitido.

\section{FORMAS DE APLICACIÓN DE RADIOFRECUENCIA}

En el tratamiento del dolor existen básicamente 2 tipos de aplicación de la energía de radiofrecuencia atendiendo a la cantidad de polos que intervienen en el proceso: monopolar y bipolar.

\section{Radiofrecuencia monopolar}

Se define la aplicación monopolar como aquella en la que el sistema está formado por un aplicador, que posee una única punta o polo activo, y que cierra el circuito eléctrico mediante una placa de dispersión (Fig. 1). 
En este tipo de configuración la densidad de corriente del campo eléctrico generado se concentra alrededor de la zona o polo activo, y se dispersa de manera rápida, siguiendo patrones distintos según nos encontremos en la punta (tip) o en el centro (shaft) de dicha punta (Fig. 8), hacia el tejido circundante de la punta/polo activo, y a su vez hacia la placa de dispersión $(12,13)$ conectada con el generador, donde el efecto térmico es nulo. Por lo tanto, la lesión térmica en el caso de CRF, o el campo eléctrico depositado en el caso de PRF crea morfologías con formas tridimensionales similares a la punta o polo activo (14-16), como veremos más adelante.

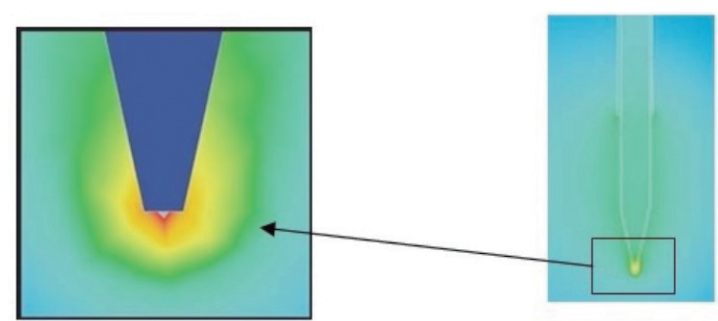

Fig. 8. Gráfico de distribución de campo eléctrico alrededor de la punta activa de la aguja, en configuración monopolar. Se puede observar que el extremo distal (tip) es el punto de mayor densidad de campo eléctrico, y que en el trayecto lateral (shaft) la densidad de campo eléctrico es menor.

\section{Radiofrecuencia bipolar}

Se define radiofrecuencia bipolar como el sistema formado por dos polos activos, y donde no interviene la placa de dispersión. El circuito eléctrico se cierra en este caso entre los dos polos activos. En este tipo de configuración, la densidad de corriente del campo eléctrico generado se concentra entre los polos activos, creando áreas de lesión mayores que en la configuración monopolar. Estas lesiones son denominadas "strip lesion". Las morfologías creadas en este tipo de radiofrecuencia dependen de varios factores (14-16).

\section{Definición de dual}

El fabricante Neurotherm (Wilmington, MA, USA) ha introducido el concepto de aplicación dual para el caso bipolar donde las puntas/polos activos no se encuentran alojados en el mismo aplicador. Por ejemplo, la utilización de dos agujas de radiofrecuencia en forma bipolar que pueden ser ubicadas a elección del operador exigiría la elección de un modo "dual". Sin embargo, cuando los polos activos se encuentran fijos en un aplicador, sin posibilidad de modificar la posición geométrica entre ellos por parte del facultativo, hablamos de configuración bipolar.

\section{MORFOLOGÍA DE LAS LESIONES}

Existen diversos estudios previos (14-16) donde se define cuál es la morfología de las lesiones creadas en las técnicas de radiofrecuencia para el tratamiento del dolor. La mayoría de ellos han abordado el carácter térmico de la lesión, tanto en configuración monopolar como en bipolar. En resumen, se puede concluir que hay ciertos parámetros clave que intervienen en el tamaño y morfología de la lesión, como son el diámetro de la aguja, el tamaño de la punta activa (también llamado longitud de la zona expuesta), el tiempo de tratamiento y, finalmente, el espacio inter-electrodo y la posición relativa entre electrodos en el caso de configuración bipolar.

Desde los primeros estudios se demostró que en configuraciones monopolares, durante la creación de una lesión térmica, el tamaño de la lesión crece en mayor medida en la zona central de la punta activa, también llamado shaft (hasta $10 \mathrm{~mm}$, dependiendo del diámetro de la aguja), que en el extremo del polo activo, también llamado tip (donde únicamente crece entre 2 y $3 \mathrm{~mm}$ ).

Esta morfología es debida a que el campo eléctrico depositado en el tejido decae proporcionalmente con la distancia $r$ recorrida desde la superficie del electrodo a razón de $1 / r^{2}$ en el tip (asumiendo idealmente el bisel de la aguja como una pequeña esfera). Lo cierto es que el valor del campo eléctrico en el tip es de gran intensidad en comparación con el del shaft, pero decae rápidamente, lo que produce que las lesiones sean muy poco extensas en la zona que rodea el tip.

Por el contrario, en el shaft, el campo eléctrico depositado decae a razón de $1 / r$ (asumiendo el shaft como un cilindro). El valor del campo eléctrico depositado en los alrededores del shaft es menor que en la zona del tip, pero tiene mayor alcance, motivo por el cual se crean lesiones mayores en esta zona.

En la práctica clínica habitual, y guiados por nuevos abordajes dictados por algunas sociedades científicas, existe una tendencia a posicionar la punta o polo activo de la aguja de radiofrecuencia lo más tangencial posible al trayecto del nervio a lesionar, para poder así crear mayores lesiones en los nervios a tratar, ya que, tal y como se comenta en el párrafo anterior, la lesión producida es mayor en tamaño en el shaft.

El tiempo de tratamiento es otro de los parámetros que condiciona el tamaño de la lesión, pero hay que tener en cuenta que, a partir de los 60-90 segundos de aplicación de la $\mathrm{RF}$, el crecimiento es mínimo respecto del crecimiento experimentado hasta ese momento. El valor límite al cual puede llegar la temperatura medida en el polo activo y la estrategia de modulación del generador cuando se da tal circunstancia, no permiten el crecimiento más allá de la morfología resultante (Figs. 9-11) hasta una isoterma de lesión térmica a $45{ }^{\circ} \mathrm{C}$, donde se puede considerar zona ablativa, con el fin de no alcanzar temperaturas de ebullición $\left(>90^{\circ} \mathrm{C}\right)$ y crear fenómeno de carbonización en la lesión.

En cuanto a la radiofrecuencia pulsada, los patrones de campo eléctrico son idénticos al caso de la radiofrecuen- 


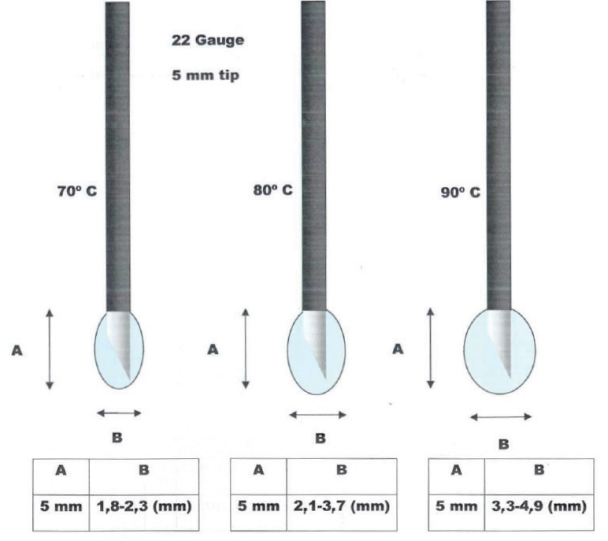

Fig. 9. Esquema de la morfología de la lesión térmica para aguja de calibre $22 \mathrm{G}, 5 \mathrm{~mm}$ de punta activa, y temperatura objetivo de $70{ }^{\circ} \mathrm{C}, 80^{\circ} \mathrm{C}$ y $90^{\circ} \mathrm{C}$. Tiempo de lesión de 90 segundos.

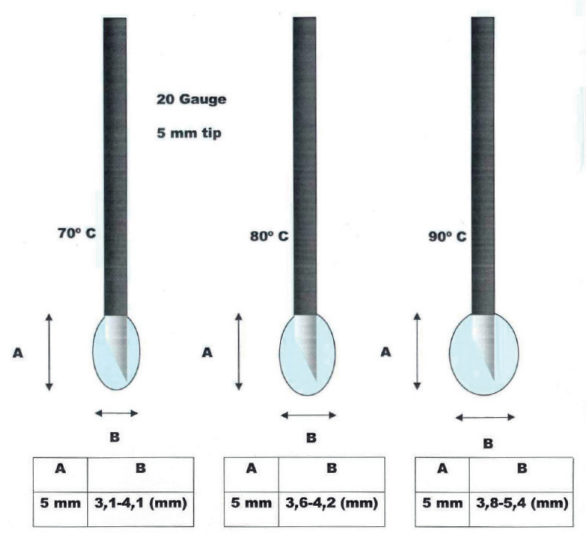

Fig. 10. Esquema de la morfología de la lesión térmica para aguja de calibre $20 \mathrm{G}, 5 \mathrm{~mm}$ de punta activa, y temperatura objetivo de $70{ }^{\circ} \mathrm{C}, 80^{\circ} \mathrm{C}$ y $90{ }^{\circ} \mathrm{C}$. Tiempo de lesión de 90 segundos.

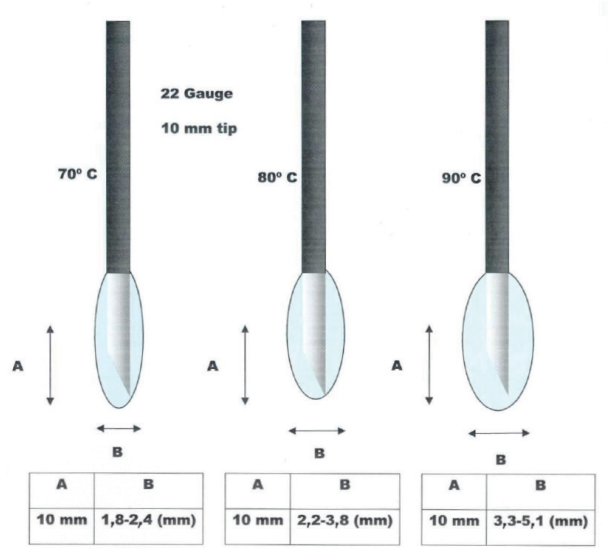

Fig. 11. Esquema de la morfología de la lesión térmica para aguja de calibre $22 \mathrm{G}, 10 \mathrm{~mm}$ de punta activa, y temperatura objetivo de $70{ }^{\circ} \mathrm{C}, 80^{\circ} \mathrm{C}$ y $90{ }^{\circ} \mathrm{C}$. Tiempo de lesión de 90 segundos. cia térmica, pero únicamente están presentes durante el tiempo en el que el pulso es emitido. Durante ese momento, y debido a los mayores valores de voltajes usados en PRF (en torno a 45 voltios), se produce un aumento de la temperatura en ese mismo instante que seguidamente es disipado en el periodo silente a través de la propia aguja y del tejido circundante. Sin embargo, existe un valor de temperatura acumulada, que es monitorizado por el generador de radiofrecuencia a través del sensor de temperatura del polo activo. Como se ha comentado anteriormente, existe una temperatura límite en modo de PRF con objeto de no crear lesión térmica o ablativa. El tiempo de respuesta de los sensores de temperatura existentes en los electrodos de radiofrecuencia comercializados actualmente no permite registrar los "spikes" de temperatura, que son pulsos de altos valores de temperatura y corta duración de tiempo que se producen en el tip de la aguja de radiofrecuencia durante el tiempo que dura el pulso. Estos "spikes" podrían ser causantes de producir minúsculas lesiones ablativas en la zona del tejido que rodea el tip de la aguja. Se han registrado valores de "spikes" de hasta $20^{\circ} \mathrm{C}$ por encima de la temperatura base, dependiendo de la distancia inter-electrodo (en el caso bipolar) y del diámetro de la aguja.

Las últimas investigaciones $(15,17)$ vinculan directamente los spikes de temperatura a la duración del pulso emitido. Se requieren mayores estudios para poder definir con exactitud en qué medida afectan los parámetros programados a los spikes y a la magnitud de campo eléctrico depositado.

En cuanto a la tendencia de colocación de la punta activa de forma tangencial o perpendicular al trayecto del nervio a tratar, parece no existir un patrón establecido a diferencia de la radiofrecuencia térmica. Los valores de campo eléctrico depositado en el tip de la aguja son muy altos respecto a los valores medidos en el shaft. Algunos autores postulan el posicionamiento perpendicular al trayecto del nervio, ya que los altos valores de campo eléctrico obtenido en el tip de la aguja podrían ser los causantes de la inhibición del estímulo nociceptivo. En este caso, posibles efectos de electroporación podrían estar produciéndose en zonas extremadamente pequeñas y circundantes al tip debido a los altos valores de campo eléctrico depositado (> $150 \mathrm{kV} / \mathrm{m}$ ). Por el contrario, otros autores aconsejan la posición tangencial del polo activo respecto al nervio a tratar. En este caso, los valores de campo eléctrico depositado son menores, pero de mayor extensión. Dicho rango de valores podría estar vinculado a posibles modificaciones en la expresión génica de indicadores relacionados con el proceso del dolor (18).

En lo referente a la morfología de las lesiones de radiofrecuencia térmica bipolar, existen varios estudios que determinan cuál es el espacio inter-electrodo máximo, en colocación espacial paralela, dependiendo del diámetro de aguja utilizado. A la vista de dichos estudios, en cualquier caso siempre en tejido ex vivo, se pueden llegar a obte- 
ner lesiones homogéneas con un espacio inter-electrodo de hasta $20 \mathrm{~mm}$, utilizando agujas de diámetro $18 \mathrm{G}$. Hay que tener en cuenta que en la práctica clínica existen ciertos factores que pueden alterar estos resultados, tales como la propia disipación térmica del tejido debido a la perfusión sanguínea y la falta de homogeneidad del tejido que podría producir irregularidades en la morfología de la lesión, siempre tomando en cuenta la mayor de las distancias inter-electrodo definida en la literatura.

Existe una tendencia actual hacia a la realización de lesiones bipolares en ciertas aplicaciones de PRF para el tratamiento del dolor. Las conclusiones de un estudio $e x$ vivo con CRF bipolar están siendo utilizadas para sugerir distancias inter-electrodo óptimas en procedimientos de PRF bipolar. Sin embargo, un estudio reciente basado en modelización matemática valoró cuál es la morfología de las lesiones térmicas creadas con esta configuración (15). Las conclusiones de dicho estudio fueron importantes en cuanto a la posibilidad real de obtención de lesión térmica en configuraciones bipolares donde la distancia inter-electrodo, en colocación espacial paralela, fuera menor a $5 \mathrm{~mm}$. Otra de las conclusiones fue la posibilidad de obtención de una zona completa de daño térmico en configuraciones bipolares con distancias inter-electrodo de $5 \mathrm{~mm}$, siempre que no actuara la limitación de temperatura (Fig. 12). En conclusión, los patrones definidos para las configuraciones bipolares de CRF no deberían ser utilizados para PRF bipolar.

Dos estudios $(19,20)$ recientes determinan el efecto de la infusión de volumen en el tamaño de la lesión en CRF
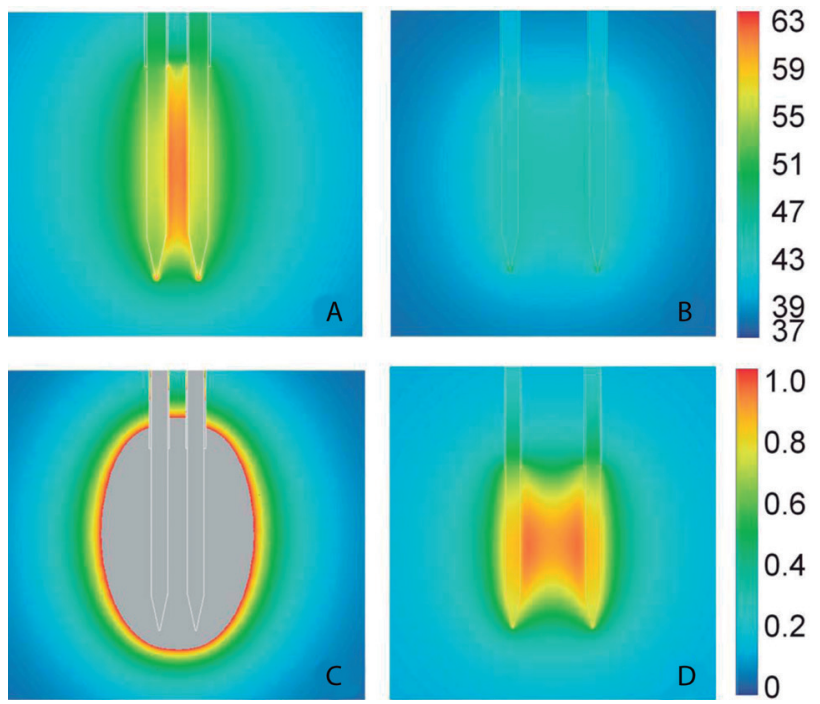

Fig. 12. Distribución de la temperatura, bajo modelización matemática, en modelo de pulsada bipolar (6 minutos, $20 \mathrm{~ms}$ de ancho de pulso, $2 \mathrm{~Hz}$ y $45 \mathrm{~V}$ ). La distancia inter-electrodo en (A) y (C) es de $2 \mathrm{~mm}$, mientras que la distancia inter-electrodo en (B) y (C) es de $5 \mathrm{~mm}$. Las figuras (C) y (D) muestran la zona de daño celular. con configuraciones monopolar y bipolar. En estos estudios se concluye que la presencia de fluidos, especialmente los que contienen electrólitos, puede inducir a la creación de tamaños de lesiones mayores, y especialmente en configuraciones bipolares. Sin embargo, una acumulación excesiva de fluido puede producir una alta conductividad, por el efecto del descenso de la impedancia, y producir efectos de coagulación de tejido en ciertos puntos demasiado cercanos al tip de la aguja, no permitiendo que crezca la lesión en su morfología habitual.

\section{ASPECTOS ELÉCTRICOS DE LA RADIOFRECUENCIA}

Las ecuaciones que gobiernan el proceso de un tratamiento de radiofrecuencia son dos: las ecuaciones de Maxwell y la ecuación de transferencia térmica en tejidos biológicos (bioheat equation) $(12,15,17)$. La ecuación de Maxwell es utilizada para el cálculo del vector de campo eléctrico $E$ que se deposita en el tejido circundante a la punta o polo activo, tomando este como un medio conductivo dieléctrico. El campo eléctrico depositado produce corrientes eléctricas en los iones de los electrolitos del tejido circundante, que se traducen en el vector $J$ de densidad de corriente que se difunde a través del tejido con la siguiente expresión matemática $J=\sigma E$, donde $\sigma$ es valor de conductividad eléctrica del tejido (medida en $\mathrm{S} / \mathrm{m}$ ). A las frecuencias base de trabajo en esta aplicación de radiofrecuencia para el tratamiento del dolor, es decir, valores próximos a $500 \mathrm{kHz}$, el medio dieléctrico próximo a la punta o polo activo se puede considerar puramente resistivo y despreciablemente capacitivo. Por lo tanto, se desprende que el vector de deposición de campo eléctrico es:

$$
P=\frac{1 / 2|J|^{2}}{\sigma}=\frac{1}{2} \sigma|E|^{2}
$$

Por otro lado, existe un efecto térmico asociado a dicha deposición de campo eléctrico que, en el caso de la radiofrecuencia térmica, en mayor medida que en la pulsada, nos va a determinar cuál va a ser la morfología y tamaño de la lesión. En el caso de la radiofrecuencia pulsada, dicho efecto sigue existiendo, pero en menores valores, ya que existe un periodo silente donde se produce una disipación de la temperatura, y la aplicación de controles de temperatura límite para no sobrepasar ciertos valores de temperatura, considerados como ablativos, sin tener en cuenta el fenómeno de los "spikes" de temperatura.

Dicho efecto y su distribución se definen a través de la bioecuación de transferencia térmica (Bioheat equation), que determina cuál es la evolución de la temperatura respecto del tiempo, en un determinado punto, al aplicar una fuente de calor, concretamente el campo eléctrico depositado en dicho punto, y tomando en cuenta también cuál es el 
impacto de la perfusión sanguínea en ese mismo punto. Los modelos ex vivo no tienen en cuenta este parámetro, que actúa disminuyendo la velocidad de crecimiento del tamaño de la lesión térmica. Los modelos ex vivo se gobiernan bajo la ecuación clásica de transferencia térmica.

Por lo tanto, la bioecuación de transferencia térmica es:

$$
\rho C_{p} \frac{\partial T}{\partial t}=\frac{1}{2} \sigma E^{2}+\nabla(k \nabla T)+W_{b} C_{b}\left(T_{b}-T\right)
$$

Donde $\rho$ es la densidad del tejido $\left(\mathrm{kg} / \mathrm{m}^{3}\right)$, y $C_{p}$ es el valor de calor específico del tejido $\left(\mathrm{J} / \mathrm{kg}{ }^{\circ} \mathrm{C}\right)$. El primer término de la segunda parte de la ecuación, $1 / 2 E^{2}$, es el campo eléctrico depositado a causa de la técnica de radiofrecuencia realizada en ese momento. El segundo término de la segunda parte de la ecuación, $\nabla(k \nabla T)$ es término de conducción térmica que tiene que ver por un lado, con el gradiente térmico (debido a las diferencias de temperatura entre puntos contiguos del tejido) y, por otro con la conductividad térmica del tejido $(k)$ expresada en $\mathrm{W} / \mathrm{m}^{\circ} \mathrm{C}$. El tercer término de la segunda parte de la ecuación, $W_{b} C_{b}\left(T_{b}-T\right)$, es la pérdida de calor producida por la perfusión sanguínea, donde $W_{b}$ es la densidad de flujo de masa de la sangre $(\mathrm{kg} /$ $\left.\mathrm{m}^{3}\right), C_{b}$ es el calor específico de la sangre $\left(\mathrm{J} / \mathrm{kg}{ }^{\circ} \mathrm{C}\right)$, y $T_{b}$ es la temperatura inicial del proceso $\left(37^{\circ} \mathrm{C}\right)$. El primer paso en un análisis físico es calcular $E$, y posteriormente introducirlo en la bioheat equation para calcular $T$. La ecuación de gobierno para el cálculo de $E$, a la frecuencia base de trabajo de $500 \mathrm{kHz}$ es $E=-\nabla \Phi$, donde $\phi$ es el voltaje eléctrico. Todo el desarrollo de ecuaciones descrito puede resolverse mediante cálculo de elementos finitos, donde la introducción de los valores de las características térmicas y eléctricas de los tejidos puede realizarse de acuerdo a valores estándar reportados en la literatura. La evolución de la lesión térmica ofrece datos para el cálculo del siguiente punto a determinar.

\section{CORRESPONDENCIA}

Víctor Muñoz

Neurotherm Spain

e-mail: victor@campoysl.com

\section{BIBLIOGRAFÍA}

1. de Andrés J, Roca G, Perucho A, Nieto C, López D, Pérez Cajaraville j; Grupo de Radiofrecuencia de la Sociedad Española del Dolor. Situación actual de la radiofrecuencia en España. Rev Soc Esp Dolor 2011;18(6):351-60.
2. López-Rodríguez MA, Varela M, Camba MA. Aplicaciones de la radiofrecuencia en el tratamiento del dolor crónico benigno. Una revisión de las publicaciones de los últimos 6 años. Rev Soc Esp Dolor 2001;8:397-411.

3. De Andrés, Roca G, Perucho A, Nieto C, López D. Generadores de radiofrecuencia disponibles en el mercado español. Rev Soc Esp Dolor 2012;19(3):157-64.

4. Vatansever D, Tekin I, Tuglu I, Erbuyun K, Ok G. A Comparison of the neuroablative effects of conventional and pulsed radiofrequency techniques. Clin J Pain 2008;24(8).

5. Schianchi PM, Sluijter ME, Balogh SE. The treatment of joint pain with intra-articular pulsed radiofrequency. Anesth Pain Med 2013;3:250-5.

6. Chua NHL, Vissers KC, Sluijter ME. Pulsed radiofrequency treatment in interventional pain management: Mechanisms and potential indications: A review. Acta Neurochir 2011;153:763-71.

7. Sluijter ME, Imani F. Evolution and mode of action of pulsed radiofrequency. Anesth Pain Med 2013;2:139-41.

8. Sluijter M, Racz G. Technical aspects of radiofrequency. Pain Practice 2002;2(3):195-200.

9. Erdine S, Bilir A, Cosman ER, Cosman ER Jr. Ultrastructural changes in axons following exposure to pulsed radiofrequency fields. Pain Pract 2009;9:407-17.

10. Chua NH, Vissers KC, Sluijter ME. Pulsed radiofrequency treatment in interventional pain management: Mechanisms and potential indications - a review. Acta Neurochir 2011;153:763-71.

11. Malik K, Benzon HT. Radiofrequency applications to dorsal root ganglia: A literature review. Anesthesiology 2008;109:527-42.

12. Berjano EJ. Ablación quirúrgica. Fuentes de energía y tecnologías. Cir Cardiov 2008;15(4):375-83.

13. Berjano EJ. Theoretical modeling of epicardial radiofrequency ablation: State-of-the-art and challenges for the future. Biomed Eng Online 2006;5:24.

14. Cosman ER, Jr., Gonzalez CD. Bipolar radiofrequency lesion geometry: Implications for palisade treatment of sacroiliac joint pain. Pain Practice 2011;11(1):3-22.

15. Pérez JJ, Pérez-Cajaraville JJ, Muñoz V, Berjano E. Computer modeling of electrical and thermal performance during bipolar pulsed radiofrequency for pain relief. Medical Physics 2014;41:701-8.

16. Pino CA, Hoeft MA, Hofsess C, Rathmell JP. Morphologic analysis of bipolar radiofrequency lesions: implications for treatment of the sacroiliac joint. Regional Anesthesia and Pain Medicine 2005;30(4):335-8.

17. Cosman ER, Jr., Cosman ER, Sr. Electric and thermal field effects in tissue around radiofrequency electrodes. Pain Medicine 2005;6(6).

18. Vallejo R, Tilley DM, Williams J, Labak S, Aliaga L, Benyamin RM. Pulsed radiofrequency modulates pain regulatory gene expression along the nociceptive pathway. Pain Physician 2013;16:E601-E613.

19. Vallejo R, Benyamin RM, Tilley DM, Kelley CA, Cedeño DL. An ex vivo comparison of cooled-radiofrequency and Bipolar-radiofrequency lesion Size and effect of injected fluids. Regional anesthesia and Pain Medicine 2014;39(4).

20. Provenzano DA. Think before you inject -Understanding electrophysiological radiofrequency principles and the importance of the local tissue environment. Regional Anesthesia and Pain Medicine 2014;39(4). 